\title{
Formaldehyde induces bone marrow toxicity in mice by inhibiting peroxiredoxin 2 expression
}

\author{
GUANGYAN YU ${ }^{1}$, QIANG CHEN ${ }^{1}$, XIAOMEI LIU ${ }^{1}$, CAIXIA GUO ${ }^{2}$, HAIYING DU ${ }^{1}$ and ZHIWEI SUN ${ }^{1,2}$ \\ ${ }^{1}$ Department of Preventative Medicine, School of Public Health, Jilin University, Changchun, Jilin 130021; \\ ${ }^{2}$ Department of Hygenic Toxicology, School of Public Health, Capital Medical University, Beijing 100069, P.R. China
}

Received November 4, 2013; Accepted June 5, 2014

DOI: $10.3892 / \mathrm{mmr} .2014 .2473$

\begin{abstract}
Peroxiredoxin 2 (Prx2), a member of the peroxiredoxin family, regulates numerous cellular processes through intracellular oxidative signal transduction pathways. Formaldehyde (FA)-induced toxic damage involves reactive oxygen species (ROS) that trigger subsequent toxic effects and inflammatory responses. The present study aimed to investigate the role of $\operatorname{Prx} 2$ in the development of bone marrow toxicity caused by FA and the mechanism underlying FA toxicity. According to the results of the preliminary investigations, the mice were divided into four groups $(n=6$ per group). One group was exposed to ambient air and the other three groups were exposed to different concentrations of FA $\left(20,40,80 \mathrm{mg} / \mathrm{m}^{3}\right)$ for 15 days in the respective inhalation chambers, for $2 \mathrm{~h}$ a day. At the end of the 15-day experimental period, all of the mice were sacrificed and bone marrow cells were obtained. Cell samples were used for the determination of pathology, glutathione peroxidase (GSH-Px) activity and myeloperoxidase (MPO) activity and protein expression; as well as for the determination of DNA damage and Prx 2 expression. The results revealed an evident pathological change in the FA-treated groups, as compared with the controls. In the FA treatment group GSH-Px activity was decreased, while MPO activity and protein expression were increased. The rate of micronucleus and DNA damage in the FA-treated groups was also increased and was significantly different compared with the control, while the expression of Prx 2 was decreased. The present study suggested that at certain concentrations, FA had a toxic effect on bone marrow cells and that changes in the Prx2 expression are involved in this process.
\end{abstract}

Correspondence to: Professor Zhiwei Sun, Department of Preventive Medicine, School of Public Health, Jilin University, 1163 Xinmin Street, Changchun, Jilin 130021, P.R. China

E-mail: zwsun@hotmail.com

Key words: formaldehyde, glutathione peroxidase, myeloperoxidase, DNA, peroxiredoxin 2

\section{Introduction}

Formaldehyde (FA) is an environmental agent commonly found in numerous products, including paint, cloth and exhaust gas, as well as other medicinal and industrial products. FA exposure has raised significant concerns due to mounting evidence suggesting its carcinogenic potential and severe effects on human health (1). Epidemiological and experimental data have demonstrated that FA may cause leukemia, particularly myeloid leukemia; however, the mechanism underlying this effect remains unclear (2-4). In the process of tumor formation, DNA damage may be the initiating factor (5), while myeloperoxidase (MPO) activity is associated with carcinogen metabolism, DNA repair inhibition and DNA damage (6-8). MPO is a specific neutrophil marker and is able to catalyze the generation of reactive oxygen species (ROS). ROS comprises a class of ubiquitous molecules that include superoxide anion $\left(\mathrm{O}_{2}\right)$, hydrogen peroxide $\left(\mathrm{H}_{2} \mathrm{O}_{2}\right)$, the hydroxyl radical $(\mathrm{OH})$ and $\mathrm{ROOH}$. When ROS formation and the levels of protective anti-oxidants are unbalanced, excess ROS causes toxic effects and ultimately leads to pathological damage (9-10).

Oxidative stress is considered to be a critical mediator of damage induced by FA, as has been observed in a number of studies (11). Peroxiredoxins2 (Prx2), a member of the Prxs family, exhibits an important role in scavenging reactive oxygen species in the body and protecting cells from damage (12). A previous study identified that Prx 2 deficiencies are associated with the onset of oxidative stress and are involved in the removal of $\mathrm{H}_{2} \mathrm{O}_{2}$ (13). Although toxicity studies of FA have been widely reported, few studies have examined the changes in Prx 2 protein expression. Therefore, the present study aimed to examine the possible toxic mechanism induced by FA and investigate whether $\operatorname{Prx} 2$ is implicated in FA-induced bone marrow toxicity.

\section{Materials and methods}

Reagents. FA (36.5-38\% in water, FW 30.03) was obtained from Beijing Jinhui Chemical Plant (Beijing, China). GSH-Px and MPO Assay kits were purchased from Nanjing Jiancheng Biotechnology Institute (Nanjing, China). The anti-MPO antibody was purchased from Santa Cruz Biotechnology, Inc. (Santa Cruz, CA, USA). The anti-Prx2 antibody was purchased 
A



Control



Control

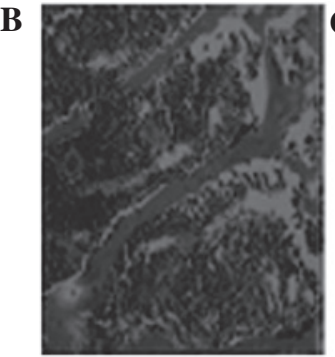

$20 \mathrm{mg} / \mathrm{m}^{3}$

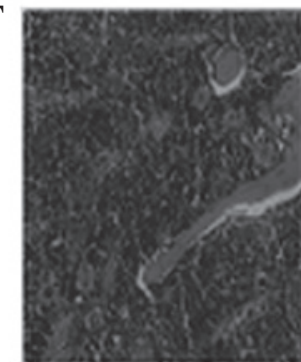

$20 \mathrm{mg} / \mathrm{m}^{3}$

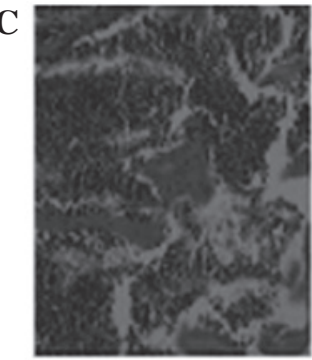

$40 \mathrm{mg} / \mathrm{m}^{3}$

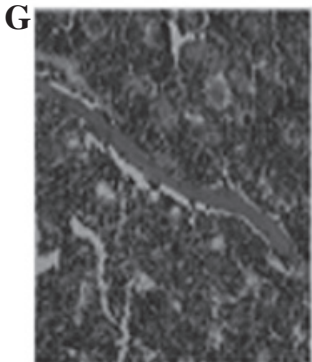

$40 \mathrm{mg} / \mathrm{m}^{3}$

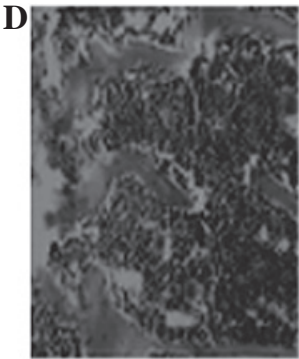

$80 \mathrm{mg} / \mathrm{m}^{3}$

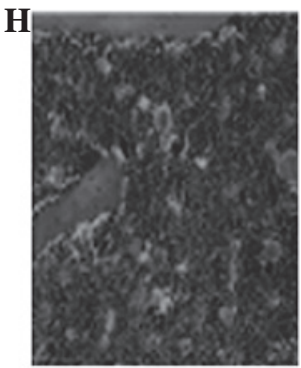

$80 \mathrm{mg} / \mathrm{m}^{3}$

Figure 1. Pathological changes of bone marrow following formaldehyde treatment in (A-D) distal and (E-H) central regions (x200). It was observed that the numbers of bone marrow cells in distal and central areas were decreased compared with the control and accompanied by sinus expansion.

from SAB (College Park, MD, USA). Agarose and ethidium bromide were purchased from Ameresco LLC (Solon, OH, USA). All reagents were of the highest purity commercially available.

Animals and treatment. Male ICR mice, weighing $13 \pm 1 \mathrm{~g}$, were purchased from the Center for Experimental Animals of the Norman Bethune College of Jilin University, (Changchun, China; Animal Certificate no. SCXK-Ji 2007-0003) and maintained under standard housing conditions (temperature, $20 \sim 24^{\circ} \mathrm{C}$; relative humidity, 40 70\%; light/dark cycle, $12 \mathrm{~h}: 12 \mathrm{~h})$. Food and water were provided ad libitum. In accordance with the principle of randomization, the mice were divided into four groups ( $n=6$ per group). According to the results of the preliminary experiment, one group was exposed to ambient air and the other three groups were exposed to different concentrations of FA $\left(20,40,80 \mathrm{mg} / \mathrm{m}^{3}\right)$ for 15 days in the respective inhalation chambers, for $2 \mathrm{~h}$ a day. All animal experiments were treated according to the procedures evaluated and approved by the Ethical Committee of Norman Bethune Department of Medicine, Jilin University (Jilin, China). At the end of the 15-day experimental period, all of the mice were sacrificed by cervical dislocation and the bone marrow cells were obtained. A number of the bone marrow cells were used for the determination of pathology, glutathione peroxidase (GSH-Px) activity, and MPO activity and protein expression; others were used for the determination of DNA damage and the changes in Prx2 expression.

Histopathology of bone marrow. The sternums of mice were extracted once the mice had been sacrificed. The sections were fixed in $10 \%$ FA solution and dehydrated and embedded in paraffin. Finally $5-\mu \mathrm{m}$ sections were cut and stained with hematoxylin-eosin. The sections were observed under a $\mathrm{BH}-2$ light microscope (Olympus, Tokyo, Japan).
Determination of GSH-Px and MPO activity. The femurs were extracted, and the surrounding muscles and connective tissues were removed. The two ends of the femurs were cut and the bone marrow cells were washed out with $0.01 \mathrm{~mol} / \mathrm{l}$ phosphate-buffered saline using a $1 \mathrm{ml}$ syringe. The bone marrow cells were filtered through 200 mesh nylon nets to obtain a single bone marrow cell suspension. GSH-Px and MPO activity was detected by MPO Activity Assay kits purchased from Nanjing Jiancheng Biotechnology Institute (Nanjing, China).

Determination of MPO and Prx2 protein expression by immunohistochemistry. The sternums of mice were removed. The bone marrow cells in the sternums were washed out and conventionally smeared. The slides were naturally dried and fixed with $10 \%$ formalin buffer solution for $15 \mathrm{~min}$, naturally dried again and placed in a $-20^{\circ} \mathrm{C}$ freezer. Next, the expression of MPO and Prx 2 proteins was detected by immunohistochemistry. Finally, the bone marrow cells were observed under a light microscope. Cells with brown particles deposited on their membrane or nucleus were counted as positive cells and the rate of positive cells was calculated using the following formula: Rate of positive cells $(\%)=$ number of cells positive for MPO or Prx 2 / total number of cells x 100.

Determination of micronuclei formation. First the sternums of the mice were removed, the bone marrow cells were washed out and then smeared onto the slides. Next, the slides were fixed and stained with a 1:9 Giemsa phosphate buffer for $15 \mathrm{~min}$. Excess dye was washed off and the slides were dried. The slides were observed under a light microscope and 1,000 polychromatic erythrocytes from each mouse were counted.

Determination of DNA damage. Single cell gel electrophoresis (SCGE) assay, also termed the comet assay was used 


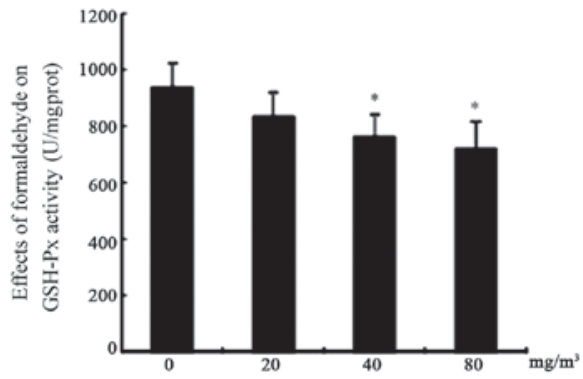

Figure 2. Effects of formaldehyde on the GSH-Px activity of bone marrow cells. Data are expressed as the mean \pm standard error. ${ }^{*} \mathrm{P}<0.05$, compared with the control. GSH-Px, glutathione peroxidase
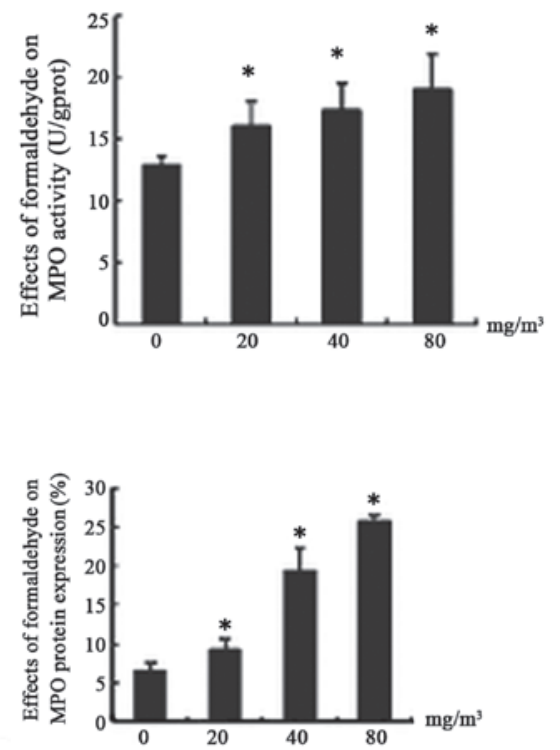

Figure 3. Effects of formaldehyde on MPO activity and protein expression in bone marrow cells. Data are expressed as the mean \pm standard error. ${ }^{*} \mathrm{P}<0.05$, compared with the control. MPO, myeloperoxidase.

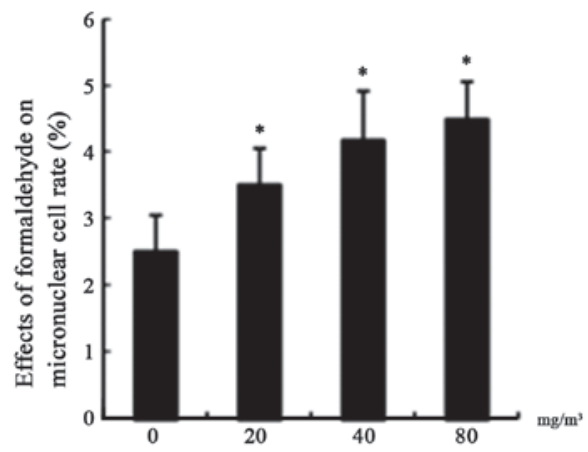

Figure 4. Effects of formaldehyde on the rate of micronucleus formation of bone marrow cells. Data are expressed as the mean \pm standard error. ${ }^{*} \mathrm{P}<0.05$, compared with the control.

to determine the DNA damage of bone marrow cells (14). Following staining by ethidium bromide (EB), the bone marrow cells were observed under a BX51 fluorescence microscope (Olympus). According to the level of damage, the DNA damage was divided into five grades, 0 , I, II, III and IV. The rate of DNA damage $(\%)=$ number of cells with damaged DNA/total number of cells x 100 (15).



Figure 5. Effects of formaldehyde on Prx 2 protein expression detected by immunohistochemistry in bone marrow cells. Data are expressed as the mean \pm standard error. " $\mathrm{P}<0.05$, compared with the control. Prx 2 , peroxiredoxin2.

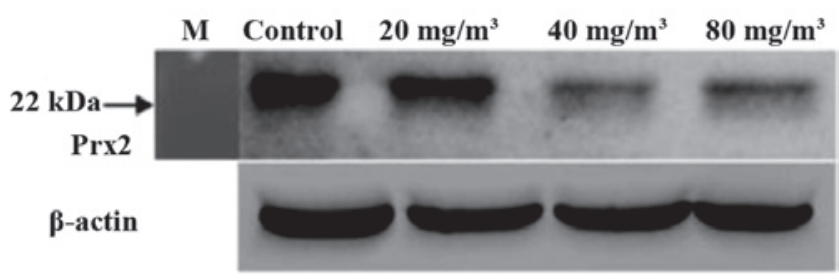

Figure 6. Western blot analysis was performed to study the expression changes of Prx 2 protein in bone marrow cells of mice. The mice were divided into control and three formaldehyde-treated groups $\left(20,40,80 \mathrm{mg} / \mathrm{m}^{3}\right)$ for 15 days in an inhalation chamber, then the expression changes of Prx 2 protein in bone marrow cells were measured. The protein expression gradually decreased as the formaldehyde concentration increased. Prx 2, peroxiredoxin 2 .

Western blot analysis of Prx 2 protein. After the single bone marrow cell suspension was established, the protein was extracted and measured. Next, $40 \mu \mathrm{g}$ of protein was separated by $12 \%$ SDS-PAGE and electro-transferred onto nitrocellulose membranes, blocked with western blotting analysis confining fluidII (1X TBS containing 5\% skimmed milk powder and $0.1 \%$ Tween-20) for $2 \mathrm{~h}$ and incubated with primary polyclonal antibodies against $\beta$-actin at a dilution of 1:3,000 and against $\operatorname{Prx} 2$ at a dilution of 1:100 for $1 \mathrm{~h}$ at room temperature. The membrane was washed and the primary antibodies were incubated for $40 \mathrm{~min}$ at room temperature with the appropriate horseradish peroxidase-conjugated secondary antibody. The bands were visualized using a Western Luminescent Detection kit (Beijing Vigorous Biotechnology Institute, Beijing, China) on X-ray film (Fusion FX7, Vilber Lourmat, Marne La Vallée, France).

Statistical analysis. Statistical analysis was performed by SPSS 14.0 statistical software (SPSS, Inc., Chicago, IL, USA). The difference between the control and FA-treated groups was tested by one-way analysis of variance and the DNA (measured by single cell gel electrophoresis assay) was tested by a $\chi^{2}$-test. $\mathrm{P}<0.05$ was considered to indicate a statistically signficant difference.

\section{Results}

Bone marrow pathology. It was observed that the numbers of bone marrow cells in distal areas were decreased, particularly in the 40 and $80 \mathrm{mg} / \mathrm{m}^{3} \mathrm{FA}$-treated groups. In the central areas, 
Table I. Effects of formaldehyde on the DNA damage in the bone marrow of mice.

\begin{tabular}{|c|c|c|c|c|c|c|c|}
\hline \multirow{2}{*}{$\begin{array}{l}\text { FA concentration } \\
\left(\mathrm{mg} / \mathrm{m}^{3}\right)\end{array}$} & \multicolumn{5}{|c|}{ No. of cells with DNA damage } & \multirow[b]{2}{*}{ Total no. } & \multirow{2}{*}{$\begin{array}{c}\text { Damage rate } \\
(\%)\end{array}$} \\
\hline & 0 & I & II & III & IV & & \\
\hline 0 & 95 & 4 & 1 & 0 & 0 & 100 & 5.00 \\
\hline 20 & 65 & 6 & 2 & 0 & 0 & 73 & 10.96 \\
\hline 40 & 78 & 10 & 3 & 0 & 0 & 91 & $14.29^{\mathrm{a}}$ \\
\hline 80 & 76 & 18 & 3 & 1 & 0 & 98 & $22.45^{\mathrm{a}}$ \\
\hline
\end{tabular}

${ }^{a} \mathrm{P}<0.05$, compared with the control. FA, formaldehyde.

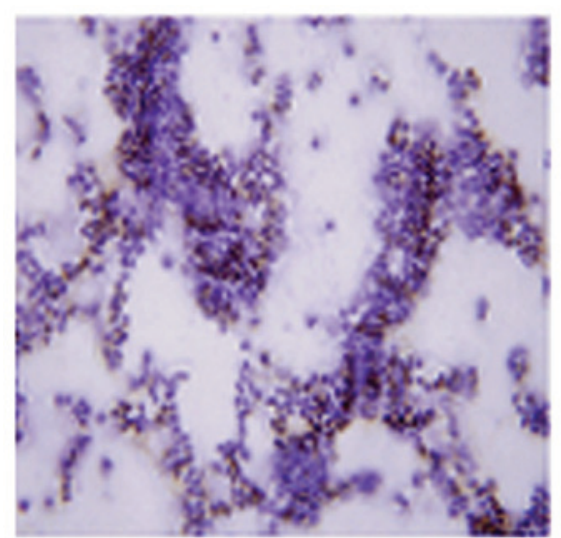

Control

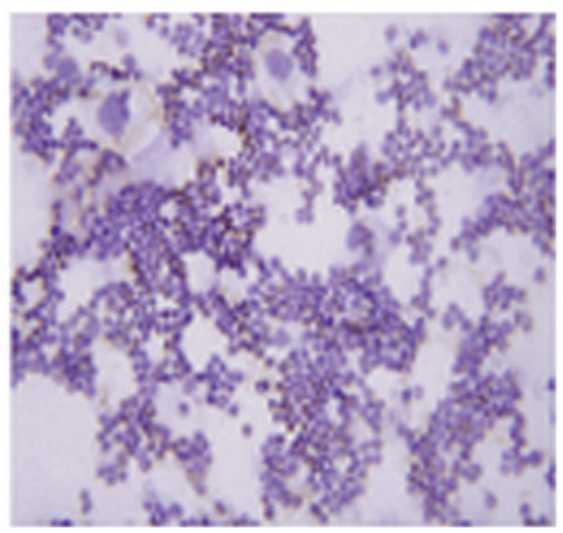

$40 \mathrm{mg} / \mathrm{m}^{3}$



$20 \mathrm{mg} / \mathrm{m}^{3}$

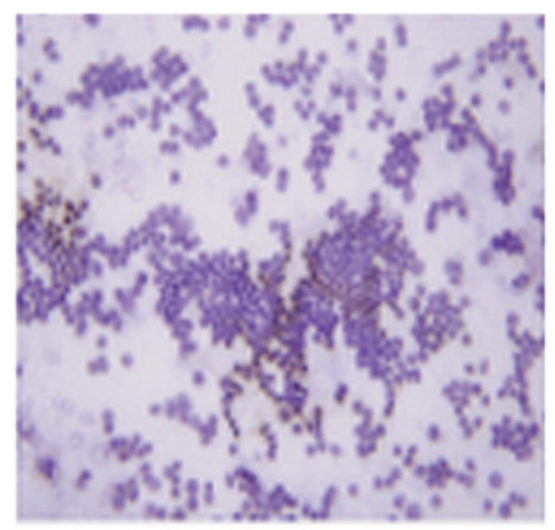

$80 \mathrm{mg} / \mathrm{m}^{3}$

Figure 7. Expression of Prx 2 protein in bone marrow cells treated with formaldehyde observed by immunohistochemistry. Brown cells are positive for Prx 2 expression (magnification, $\mathrm{x} 200$ ). Prx2, peroxiredoxin2.

the numbers of bone marrow cells were also decreased and were simultaneously accompanied by the sinus expansion. (Fig. 1).

Effects of FA on GSH-Px activity. Following FA exposure, the GSH-Px activity was significantly decreased, and there was a significant difference in the activty in the 40 and $80 \mathrm{mg} / \mathrm{m}^{3}$ FA-treated groups compared with the control ( $\mathrm{P}<0.05$; Fig. 2).

Effects of FA on MPO activity and protein expression. MPO activity and protein expression were increased in a dose-dependent manner compared with the control $(\mathrm{P}<0.05$; Fig. 3).

Effects of FA on the rate of micronucleus formation. The reate of micronucleus formation in each FA-treated group was increased and the difference was significant compared with the control ( $\mathrm{P}<0.05$; Fig. 4).

Effects of FA on DNA damage. The DNA damage of bone marrow cells was detected by SCGE, in which the DNA-damaged cells had comet tails and were divided into five 
grades according to the level of damage. The results demonstrated that the DNA damage rate was enhanced as the FA concentration increased and there was a significant difference compared with the control $(\mathrm{P}<0.05$; Table I).

Expression of Prx 2 protein. The expression of Prx 2 protein was detected by immunohistochemistry and western blotting (Figs. 5 and 6). The results demonstrated that Prx 2 expression decreased as the FA concentration increased, and there was an evident difference in FA-treated groups compared with the control $(\mathrm{P}<0.05)$. As demonstrated in Fig. 7, the number of brown positive cells progressively decreased between the FA-treated and control groups.

\section{Discussion}

FA is an electrophilic agent. As an oxidant with weak radicals, it causes chemical processes similar to the Fenton reaction and produces strong oxidants, including the hydroxyl radical. Hydroxyl radicals combine with glutathione (GSH), an important antioxidant in the body, to form thio-hemiacetal. This process causes GSH depletion damaging the redox balance in the body and resulting in oxidative stress. The process of lipid peroxidation is a type of oxidative stress, which can cause cell dysfunction by producing a variety of free radicals and non-radical products, particularly lipid free radicals. These lipid free radicals easily penetrate and diffuse into the nucleus, act on nucleic acids to induce DNA base modifications and strand breaks, and thus lead to cell mutations and cancer. Studies have demonstrated that MPO catalyzes the process of lipid peroxidation (16).

The present result demonstrated that a certain concentration of FA may decrease GSH-Px levels and increase MPO activity and protein expression. GSH-Px is a selenium-containing protein, GSH is its main substrate and binding results in conversion of harmful compounds into harmless hydroxyl compounds and water. Therefore, it is able to protect the structure and function of the cell membrane from damaging peroxide, and may effectively remove lipid peroxides from the body. MPO is an important heme-containing enzyme released by Azure particles of neutrophils and catalyses the reaction between hydrogen peroxide and chlorine to produce hypochlorous acid. Therefore, it is important for the development of host defense and inflammation. In addition, MPO is also a functional sign and an activation marker of polymorphonuclear (PMN) leukocytes, the change in MPO activity represents the function and activity status of PMNs. FA may cause GSH depletion and lead to lipid peroxidation. This process may trigger the activation of PMNs. When PMNs are activated, they are able to release MPO into the extracellular space or into phagosomes by degranulation, which stimulates $\mathrm{H}_{2} \mathrm{O}_{2}$ to generate a series of ROS with broad biological effects at sites of inflammation. In addition, MPO may also pass activated signals from the cytoplasm to the nucleus by a cell-signaling pathway to induce the expression of inflammation-related genes. The pathology results demonstrated that the number of bone marrow cells in the 40 and $80 \mathrm{mg} / \mathrm{m}^{3}$ FA-treated groups were evidently decreased and accompanied by a sinus expansion. This suggested that FA was able to cause an inflammatory reaction in the bone marrow. MPO may therefore promote this process.
Increased lipid hydroperoxide production by FA may be associated with Prx 2 activity. Prx 2, a member of the peroxidase family, acts as a key scavenger of ROS. It is able to modulate cytokine-induced $\mathrm{H}_{2} \mathrm{O}_{2}$ levels, which have been demonstrated to mediate signaling cascades leading to cell proliferation, differentiation and apoptosis. In addition, it also has an important role in peroxide detoxification in cells in cooperation with GSH-Px (17). In the present study, it was identified that the decrease in Prx 2 expression was associated with the bone marrow toxicity induced by FA. The downregulation of Prx2 increases oxidative stress (18), resulting in subsequent toxic effects and inflammatory responses. Consistent with these results, several other studies have observed that, among the six Prx isoforms, only Prx 2 expression decreased in response to FA (19).

The present study also examined the effects of FA on DNA damage, using the micronucleus test and SCGE. The results suggested that certain concentrations of FA may cause chromosome breakage and the formation of micronuclei during the process of cell division in vivo. One hypothesis is that FA interferes with spindle fiber function or exhibits a role in internal chromosomal bonding, which may cause chromosome breakage to form micronuclei. FA may also cause lipid peroxidation to produce lipid free radicals. These lipid free radicals may act on nucleic acids to induce DNA base modifications and strand breaks, causing an increase in micronuclei. When detecting DNA damage using SCGE, it was identified that there was also an evident difference between the FA-treated groups and control groups.

In conclusion, the results suggest that certain concentrations of FA may have toxic effects on bone marrow cells. Lipid peroxidation had an important effect and the MPO level promoted this process. It was also identified that decreased Prx 2 expression was implicated as an important mechanism underlying FA-induced bone marrow toxicity.

\section{References}

1. National Toxicology Program: Formaldehyde. Rep Carcinog 12: 195-205, 2011.

2. Cole P and Axten C: Formaldehyde and leukemia: an improbable caused relationship. Regul Toxicol Pharmacol 40: 107-112, 2004.

3. Schwilk E, Zhang L, Smith MT, Smith AH and Steinmaus C: Formaldehyde and leukemia: an updated meta-analysis and evaluation of bias. J Occup Environ Med 52: 878-886, 2010.

4. Speit G, Gelbke HP, Pallapies D and Morfeld P: Occupational exposure to formaldehyde, hematotoxicity and leukemia-specific chromosome change in cultured myeloid progenitor cells. Cancer Epidemiol Biomarkers Prev 19: 1882-1884, 2010.

5. Yingcheng Xie: The progress of DNA damage and tumor. Chin J Cancer 16: 313-317, 2006.

6. Neuss S, Holzmann K and Speit G: Gene expression changes in primary human nasal epithelial cells exposed to formaldehyde in vitro. Toxicol Lett 198: 289-295, 2010.

7. Ladeira C, Viegas S, Carolino E, et al: Genotoxicity biomarkers in occupational exposure to formaldehyde - the case of histopathology laboratories. Mutat Res 721: 15-20, 2011.

8. Klein MD, Sinha BK and Subramaniam RP: Statistical inferences from formaldehyde DNA-protein cross-link data: improving methods for characterization of uncertainty. J Biopharm Stat 21: 42-55, 2011

9. Zhou DX, Qiu SD, Zhang J, Tian H and Wang HX: The protective effect of vitamin E against oxidative damage caused by formaldehyde in the testes of adult rats. Asian J Androl 8: 584-588, 2006.

10. Kovacic P and Somanathan R: Dermal toxicity and environmental contamination: Electron transfer, reactive oxygen species, oxidative stress, cell signaling, and protection by antioxidants. Rev Environ Contam Toxicol 203: 119-138, 2010. 
11. Zhang Y, Liu X, McHale C, et al: Bone marrow injury induced via oxidative stress in mice by inhalation exposure to formaldehyde. PLoS One 8: e74974, 2013.

12. Cox AG, Winterbourn CC and Hampton MB: Mitochondrial peroxiredoxin involvement in antioxidant defence and redox signalling. Biochem J 425: 313-325, 2010.

13. Smith-Pearson PS, Kooshki M, Spitz DR, et al: Decreasing peroxiredoxin II expression decreases glutathione, alters cell cycle distribution, and sensitizes glioma cells to ionizing radiation and $\mathrm{H}(2) \mathrm{O}(2)$. Free Radic Biol Med 45: 1178-1189, 2008.

14. Singh NP, McCoy MT, Tice RR and Schneider EL: A simple technique for quantitation of low levels of DNA damage in individual cells. Exp Cell Res 175: 184-191, 1988.

15. Anderson D, Yu TW, Phillips BJ and Schmezer P: The effect of various antioxidants and other modifying agents on oxygen-radical-generated DNA damage in human lymphocytes in the COMET assay. Mutat Res, 307: 261-271, 1994.
16. Podrez EA, Abu-Soud HM and Hazen SL: Myeloperoxidase-generated oxidants and atherosclerosis. Free Radic Biol Med 15: 1717-1725, 2000.

17. Wood ZA, Schroder E, Robin Harris J and Poole LB: Structure, mechanism and regulation of peroxiredoxins. Trends Biochem Sci 28: 32-40, 2003.

18. Qu D, Rashidian J, Mount MP, et al: Role of Cdk5-mediated phosphorylation of Prx2 in MPTP toxicity and Parkinson's disease. Neuron 55: 37-52, 2007.

19. Lim SK,Kim JC,Moon CJ, et al: Formaldehyde induces apoptosis through decreased Prx 2 via p38 MAPK in lung epithelial cells. Toxicology 271: 100-106, 2010. 